von Sftober bis Miärz ift bie Feriobe ber ftärfften Iätigleit in ber

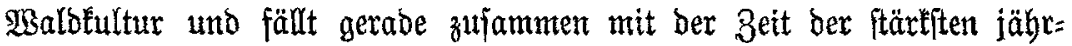

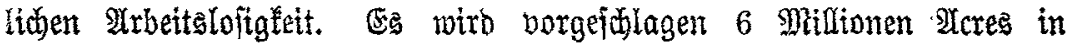
Schottland, $2^{1 / 2}$ Millionen 2 cres in England und 500000 2cres in Srland aufzuforften.

Der Beridjt Der Rommijfion mie bas \$rojeft an fick ift won ber gejamten englijden Preffe, obne Unterjüieb ber $\mathfrak{P a r t e i}$, mit ungeteiltem Beifall aujgenommen worben. Es wirb in ben jpaltenlangen Bejprechungen

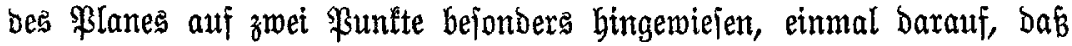

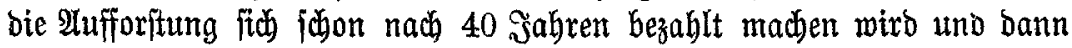
barauf, bá bie angelegten Waldungen nach 80 saburen England mebr

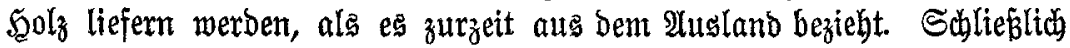
wirb bann nod) barauf hingemiejen, bá bie 2 trbeitelofenfrage, bie fith immer mehr unb mebr zu einem bunflèn Fled in bex modernen Entwictlung bes englifichen Erwerbellebens geftaltet, burch bas 2lufforitungsprojett, wann nidft ganz gelöft, fo bod in ibren bedenflidbiten (Eridgeinungen er= hebliç gemildert miro.

Sonoon, 26. Samuar.

$\mathrm{Br}$.

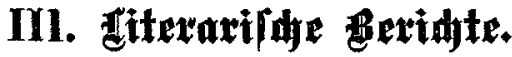

গir. 40.

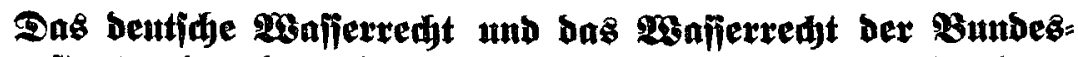

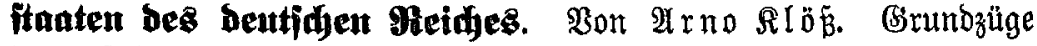

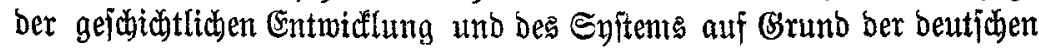

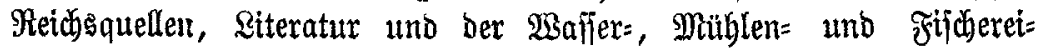
gejebgebung ber Bundesitaaten. Sgalle a. S., Berlag von Wilh. Knapp, 1908. Freis ungeb. 6,60 Mll.

Der etwas umfangreif) gehaltene Titel biejes Buches bentet bereits an, bá es fich bier in erfter sinie um eine Darftellung ber Gejejichte Des beutichen $\mathfrak{B a f f e r r e d i e s ~ u n o ~ b e r ~ e i n z e l n e n ~ B u n d e s f t a a t e n ~ h a n d e l t . ~}$

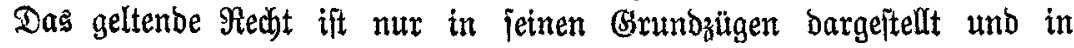
einem zweiten $\mathfrak{B u}_{\text {th }}$ veripridgt ber Berfaffer einen Rommentar aller

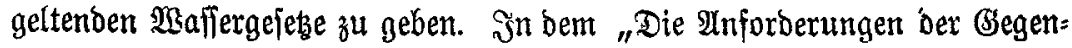

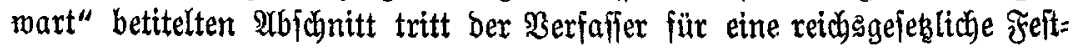

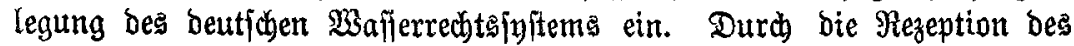

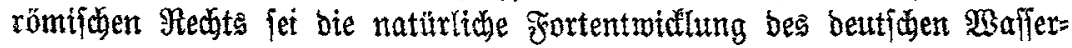

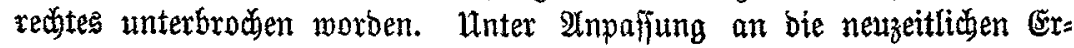




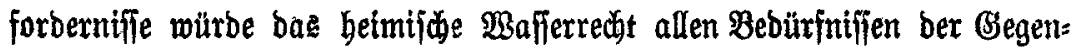
wart zu genügen vermögen. - Das Bud tit flax uno anregeno ge=

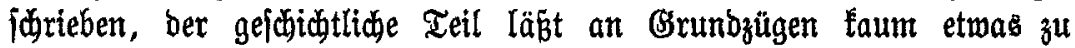
wünidben übrig. Darum ift es bantbar zu begrüben.

E.

\section{Ir. 41.}

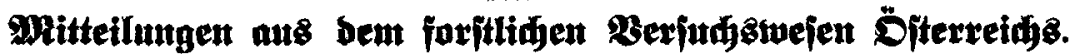

XXXIV. Şeft. Form unb Эnbalt ber Tanne. Bon Adalbert

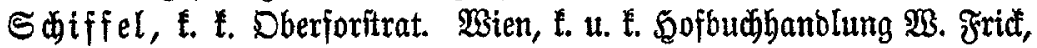
1908.

Der um bie Baum= und Beftandamaffenermittlung perbiente $\mathfrak{B e r}=$

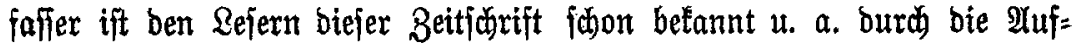
ftellung von Frorm= und Maffentafeln für F̌tchte, \&ärche uno Ritefer, benen ex itn vorliegenden Sheft bie Tanne anreibt. Damit will bie öfterreichijhe

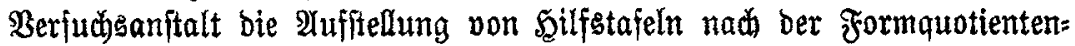

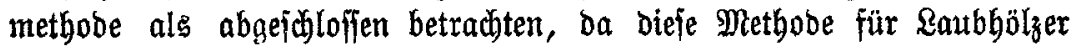
nidyt in Betradit fommen Eann.

Das Seft enthält 3 ber Mafienermittlung bienende Tafeln: I. Form= zahlen = und Formquotiententafel, bie mit bem Eingange natb Söbe uno Formquotient $q_{2}\left(=\frac{\text { Mittendurdomeffer }}{\text { Bruftböhendurdjmeffer }}\right)$ bie Shaft= und $B a u m=$ formzahl angibt für ßaumböben von 6-48 m. II. Form= und Maffen= tafel, bie mit bem Eingang Göhe, Brufthöhenourchmefier und Form= quotienten $q_{2}$ (bezm. Formflafie) Den Derbholz=, Schaft= und Bauminhalt, forie bie Durbmeffer in $1 / 4,1 / 2$ und $3 / 4$ ber Sdaftlänge angibt, woburd. zugleich eine Zerlegung ber Maffe in Sortimente ermöglidft ift. III. Maffen= und Derbholzfortierungstafel, bie gebrautbt wirb, wie eine gewöhnlide Maffentafel mit bem Eingange nad Söhe und Brufthöhenourdomefier.

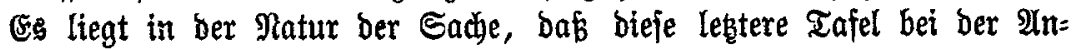
wenoung auf Einzelftämme nicht immer ein genaues Rejultat geben tann, es find 2 bromeidunger bis zu $15 \%$ möglith, und aud bei ihrer $\mathfrak{A n}=$ wentung auf Slafjenmittelftämme find nod) erbeblidłe Febler möglid.

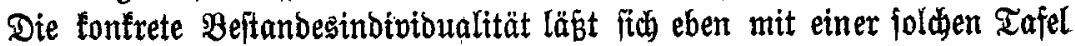

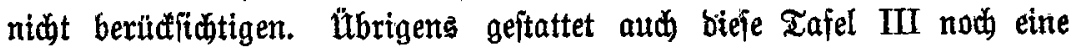
grobe Sortierung, ba fie AZngaben über Derbholz: Ränge unb Mittenitärfe enthält.

Die Tajel I und II geben, aud auf ben Cinzelftamm angewendet,

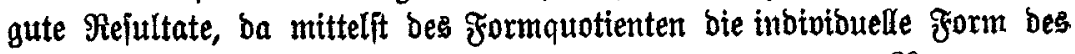


Sぬhaftes berüffithtigt wirb. 3ur Ermittlung bes Formquotienten ift bie Mefiung bes Durdimefiers in $\frac{\mathrm{h}}{2}$ notmendig. Es läbt fich aber, freilich auf Soften ber (Genauigfett, $q_{2}$ aud indireft ermitteln im 2 (nnhalt an

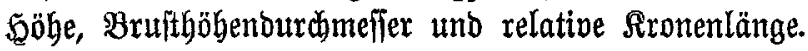

Nach) Fertigitellung ber Tajeln für bie 4 Sauptnabelyälzer lag es nabe, zu unterjuchen, $\mathfrak{b b}$ und inmiemeit fich Formen = und Snbalts= unteridjiebe ergeben. Da zeigt fid nun, bá̉ bei Schäften mit gleidjem $h, q_{2}$ und $d 1,3$ Sdhaftinfalt und Schaftformzahl ber veridiebenen

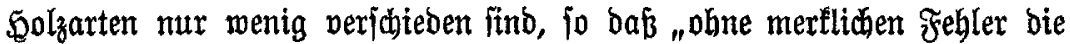

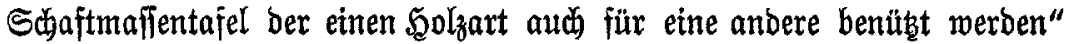
fann.

Die Schaftform bagegen weift bebeutende Serichiebenheiten auf, menn man ben $\mathfrak{B e r l a u f}$ ber Shaftfurpen in ben veridiedenen Sdaft=

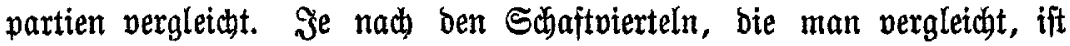
bald bie eine, bald bie anbere Art vollbolziger.

Bergleidft man bie burdiffonittlichen Frormquotienten ober bie Extreme

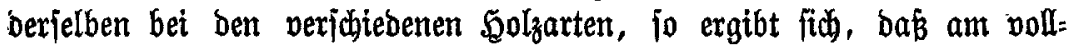

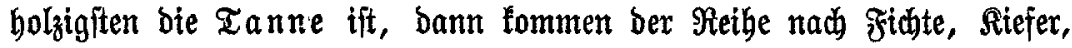
\&ärçe.

Schüp fer.

Sir. 42.

Der Deutide 23alb. Bon \$rofeĩor Dr. $\mathfrak{R}$. $\mathfrak{B}$ uesgen. Mit zabl= reiden Ubbildungen und 2 Tafeln. Reipzig, Berlag von Duelle \& Wiener. 176 S. Ellegant geb. $1,80 \mathscr{M}$.

Das vorliegende Büdlein ift ein Gitied einer von Sonrab Şöller

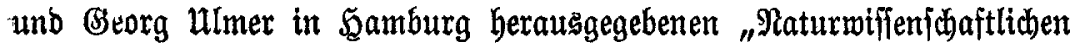

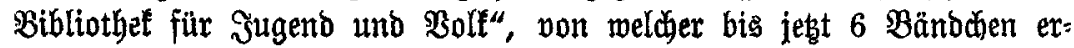
jobienen find.

Für Sugend und Bolf ift aljo bas Dem furzen Borwort bem Rejer, Dem Der Mald nach wohl nur wenig befannt ift, in leidft veritänolidjer Daritellung biejen jojilbern, ben \$̧albfreund auf mandbe Bseheimniffe aufmerfjam madben,

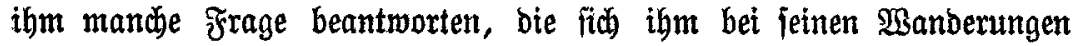
etwa aufbrängt. S̊n 12 loje aneinander gereihten $\mathfrak{X b j a b n i t t e n ~ f u ̈ b r t ~ b e r ~}$ Berfaffer ben Rejer in ben beutjhen $\mathfrak{M a l d}$ ein, jojildert $i \mathfrak{b m}$ ben Eidhen= und Butben=, ben Riefern=, Jithten= und Tannenwald in ihrer Eigenart, wirft auf ben Baumftamm uno jeine Bildung, auf bie Gewinnung von

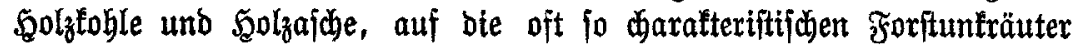

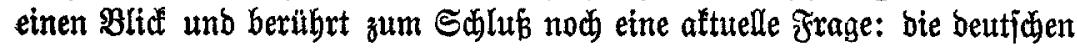


Solonialwälber. Gerne wirb ber Raie ben anregenben Sdjilberungen

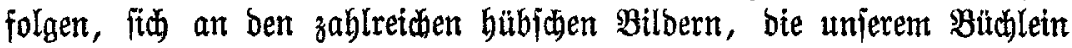
beigegeben ïnd, erfreuen und mandhe Belehrung bent lekstern entrehmen.

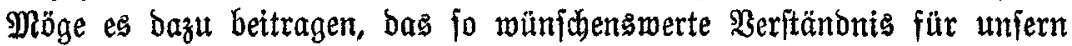

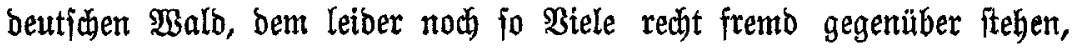
in weite Rreife unjeres $\mathfrak{B} 0$ lfes zu tragen!

F.

\section{Nr. 43.}

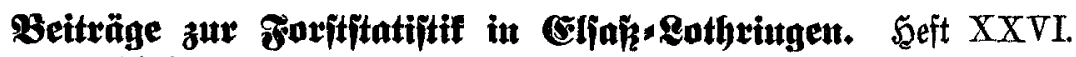

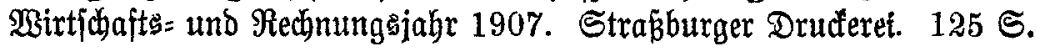
Das Birtichaitsjabr 1907, bas itth infolge ber Sodjtonjunltur gleid,

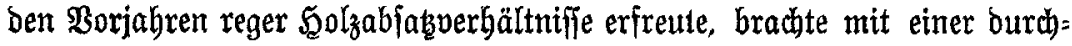
idnittlidjen 2 tonutang von $4,22 \mathrm{fm}$ Bejamtbolzmaffe im Staatsmalbe eine verbältnismääßig hohe Biffer, mit $4,64 \mathrm{fm}$ in ben Gemeinde= unto

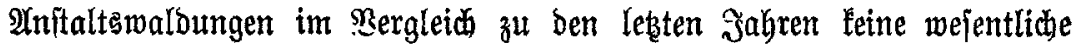

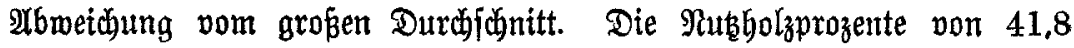
(Staatswalb) und 32 (Semteindewald) fönnen als angemeffen boc gelten.

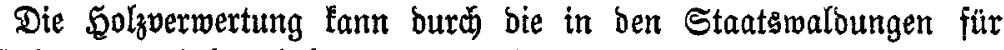
verfteigertes $50 l_{z}$ erbobenen Freigzablen in Miar ziemlich genau zum 2Us

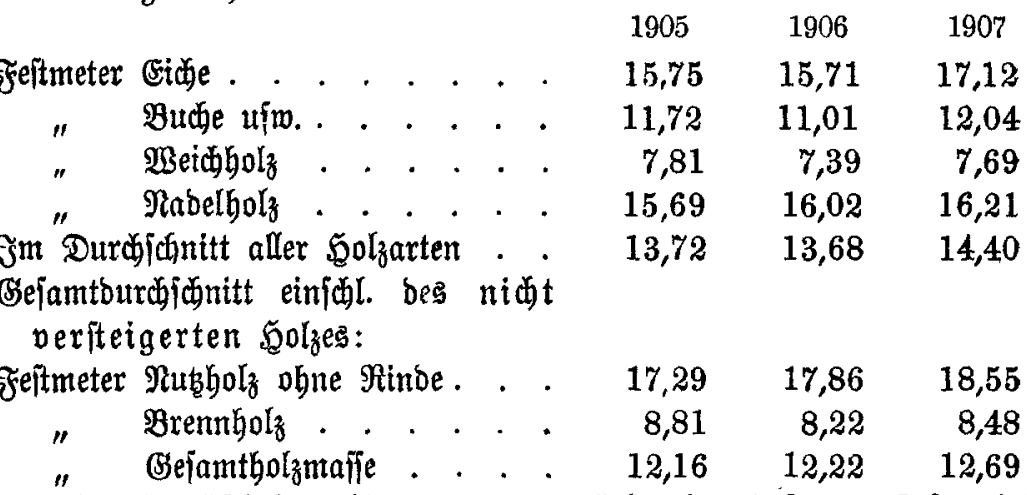

Der Durdjfobnittserlös von $12,69 \mathscr{A}$ für bas Feeftmeter Bejamtholz=


Rothringen nod) nie erreidtes Maximum, bem gegenüber bas ablaufende

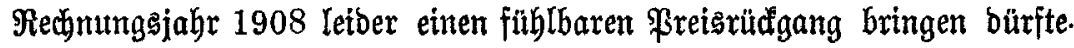

Die $\mathfrak{B r u t t o}=$ Solleinnahme für $\mathfrak{S}_{\mathrm{ol}}$ ftellte fin im Staatsmalbe gegenüber 1905 mit 7,3 Millionen Marf, 1906 mit 7,7 Millionen Marf 1907 auf 8,1 Mtllionen Marf, eine jeit 1871 mur nad Bittidafts= jahren mit abnorm erböhtem Einjolage (Winbjalljahre!) erreidte Summe. 
2luc dieje wixb 1908 vorausfthtlid füblbar zurüdgehen.

Die Flädhenabjuflüffe für bie Staats: unb ungeteilten Waloungen bebeuten mit 154781 ha und bie Gemeinbe= und $\mathfrak{A n}$ ftaltswaldungen mit 201834 ha gegenüber bem \$orjabre eine Bunabme von 322 beżw. 62 ha.

Şn biejen jämtlicben unter ber Foritoronung itzhenden 23 albungen

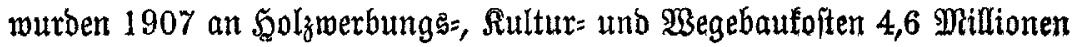
Mart verauggabt, etwa $13 \mathscr{M}$ auf bas Seftar.

$\mathfrak{Z} \mathfrak{u} \mathfrak{j} 75800$ ha verwalteter Staatswalbjagd wurben 1907 erlegt:

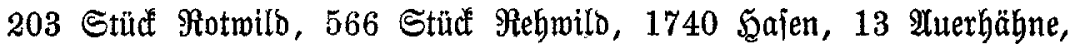
27 Stüd Birt: und 5ajelwild. $2 \mathfrak{n}$ fajäblituem $\mathfrak{B i l}$ wurben 1907 zur Strecte gebradbt: 689 Sauen, 2072 Füdje, 48 Wilbtaken. $\quad$ Rahl.

Rr. 44.

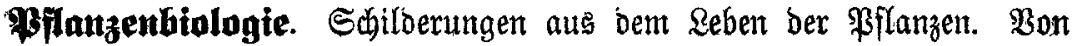
Dr. W. Migula, Profeffor an ber Forftafabemie Eifenach. Mit 133 Tertfiguren uno 8 Tajeln. Budjommute von Gedjo Beil and. Leipzig, Berlag von Duefle \& Mener, 1909. 352 ؟. \$reis geb. 8,80 A.

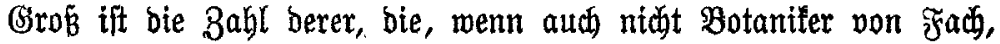
fo boch Freunde ber PIlanzenwelt find; und neben benen, bie fich in erfter Sinie für beren Form und Beitalt, für bie Syitematif intereffieren, fteht wohl eine ftets fteigenbe $3 \mathfrak{a b l}$ jener, welchen bie fo mannigialtigen

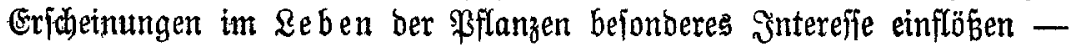
und bem Bedürinis biejer lebsteren mödte ber Berjaffer burdh bie vor= liegende "\$Flanzenbiologie" genügen. (Ex hat fid babei laut $\mathfrak{B a r m o r t}$ bemüht, aus bem gewaltigen (Gebiet bie bejonbers widtigen Erideinungen herauszagreifen, und will ntaft ein Rehrbuch ber Pflanzenbiologie, jonbern eine einfache und gemeinneritändliche Daritellung jener Eridbeinungen geben, weldhe von allgemeinerem șntereffe fino.

Einer furzen Einleitung "Die Entwicklung der Pflanzenwelt", folgt im

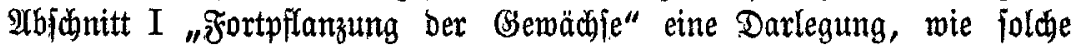

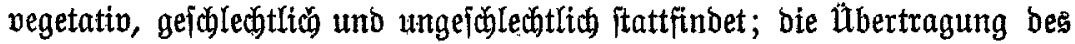

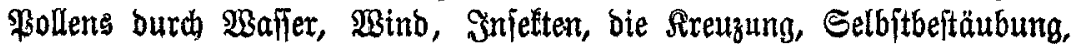
Baftaroterung fimben Erörterung.

Der II. Albfhnitt bebandelt "Die Berbreitung ber Bflanzen" und

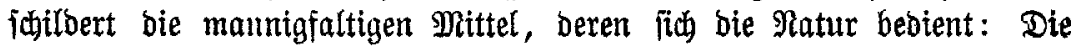

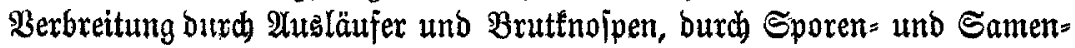
vertragung, burch $\mathfrak{B a j p e r}$, Wint, Itere jeber $\mathfrak{A r t}$, uno bie vielieitigen Borriftungen, bie biefer Bertragung bienen - ein horbinterefiantes Bebiet! 


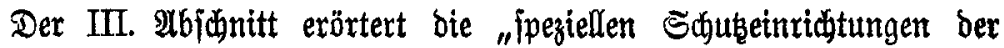
\$Flanzen“, bie benfelben gegenüber ungünftigen Rebensbebingutngen, \$ilzen,

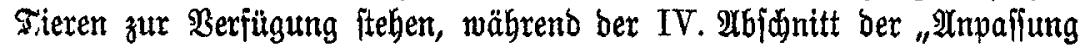

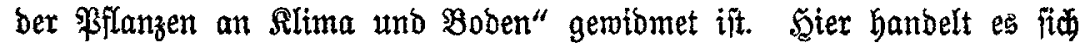
um bie $2 \mathfrak{l n p a f f u n g}$ an beftimmte Medien, an Trochenbeit unb Fentdigtigfeit,

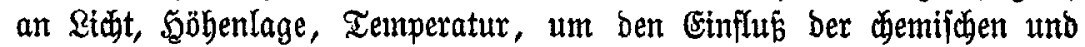
phyftitalijden $\mathfrak{B}$ ejdaffenheit des Bodens.

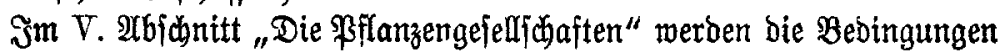

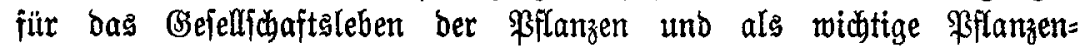
gefellichaften ber $\mathfrak{B a l d}$, bie Grasuegetationen, Saetoe und Moor be= iprocten.

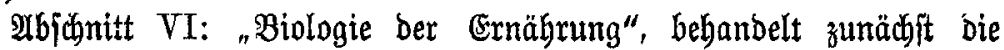

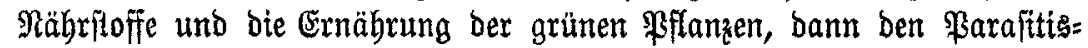
mus, bie Saprophyten, bie Mytorbiza und bas interejante Sapitel ber

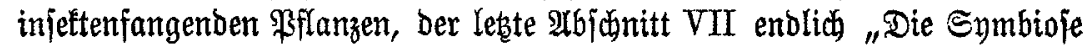
uno bas (Senofienidhaftsleben “, wie foldhes bet ben Fledten, ben Anöllden= bafterien ber Reguminojen, der Symbioje zwijden algen unb nieberen Tieren in bie Eribheinung tritt.

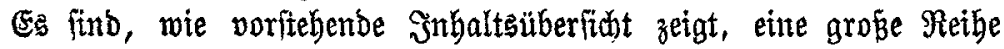
widtiger biologijder Eribeinungen, bie ber Berfaffer in hödit anziebenber Reije behandelt; zahlreide in Den Tert eingebrudte 2 Abbildungen bienen

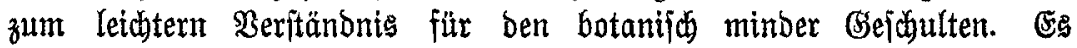
bürfte Dem Serfaffer gelungen fein, Den im Borwort getennzeichneten 3wed feiner Pflanzenbiologie in vollem $\mathfrak{M a \mathfrak { B }}$ zu erreichen und bürfte bem febr gut ausgeitatteten $\mathfrak{W e r l}$ freundithe 2 lnerfennung unb Serbreitung in weitem Sreije nic̆t fehlen!

Dr. Fैürft.

\section{Ni. 45.}

Rebensbilber aus ber Tierwelt. Gerausgegeben won $\mathfrak{S}$. Meer= warth. Zweite Folge: Bögel. Mit äber 200 photogr. Ilbbildungen.

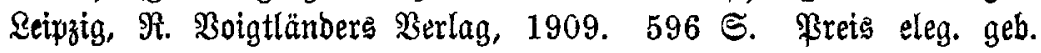
14 .h.

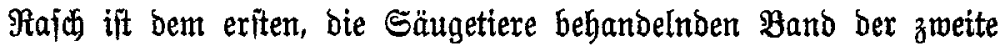
gefolgt, Der $\mathfrak{B}$ ogelwelt gewiomet. WBir baben ben eriteren im Märzheft

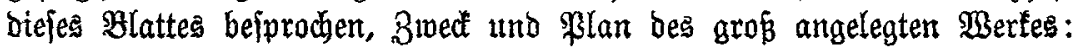
ein biologifies Tierbuch mit ausmabmslos photographifoen 2 Uufnahmen lebenber, unb zwar foweit möglidy nur frei lebenber Tiere, illuftriert zu f(u)affen - mitgeteilt, uno fönnen une biex łürzer faffen.

Ein Teil unjerex Bogelwelt - man benfe an viele Singuögel, 
Droffeln, Staare u. a. - bietet infolge feiner geringeren Scheu gegen= itber bem Teenjoden wohl bor photographifhen Ilufnahme minbere Schmierigfeiten, als unfere icheuen Bierfüpler; aber bei anderen find biefe wohl ebenjo großz, und wer fich bavon überzeugen will, ber möge bie $\mathfrak{B i l b e r}$ zu bem $24 b j$ thnitt "Der Stein= ober Golbabler" betracten, bie wohl zu bem gropartigiten gebören, was auj bem Bebiete ber Matur: aufnabmen geleiftet murbe.

Die $3^{\mathfrak{a} h \mathfrak{l}}$ ber photographijd bargejtellen und mit exläuternbem Text von veriditebenen Autoren, insbejondere von Martin $\mathfrak{B r a ̈ z}$ uno

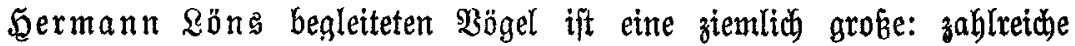

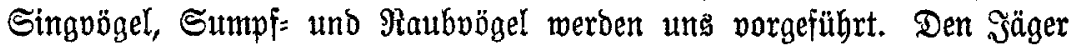

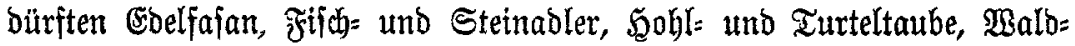

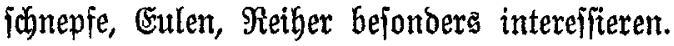

Den jebr zahlreidhen Billbern, bie je nach Bröße teils auf eigenten Tafeln, tetls in ben Tert gebructt und burchaus infolge forgfältiger

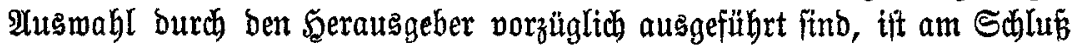

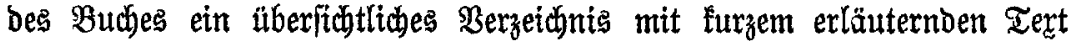
beigegeben.

Beitere Folge, die fin insbejondere auf unjere jagolth jo intereffanten

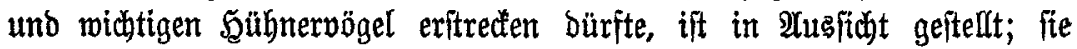
wirb gletid bem vorliegenden von bem serlag vorzüglid ausgeltatteten Band fidter warme $\mathfrak{A}$ frnahme jeitens aller Tierfreunbe finden.

Dr. Fürút.

शr. 46.

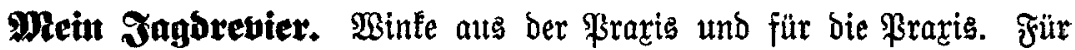
Jagbherren, Beï

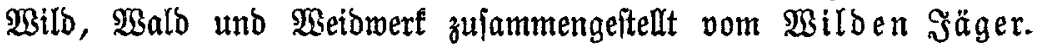

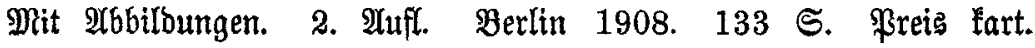
$3,75 \mathscr{M}$.

Der unter obigem Ramen in der Sägermelt wohl befannte erfahrene Weibmann hat bie ftille 3eit, während weldjer bie F̧iridje ßolben tragen und $\Re$ uhe braudten, benübt, um in jeiner Bergangentheit zu blättern und auf (Srumb jeiner langiäbrigen und vielieitigen Erfahrungen 2 Inregungen

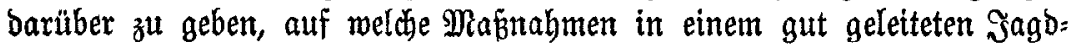

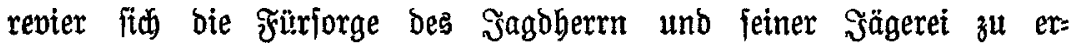
ftrecten babe.

3unäbift weift ex auj bie Borftcht hin, bie man heutzutage bei ber enormen fonfurrenz walten laffen mus bei ber Grpadtung von Sagben — von Gsemeinben, Stäbten, Grö̈grunbbefikern; fubilbert bann bie $\mathfrak{M a} \mathfrak{k}=$ 
regeln, burch bie man bie ฐago auf eigenem, wenn auth nur mäßig

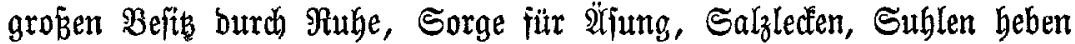

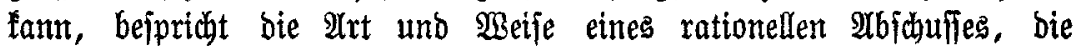

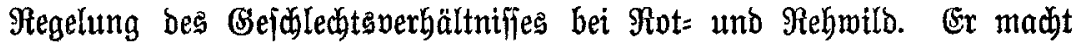

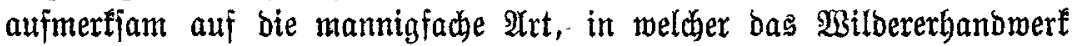
betrieben wirb, interefiante eigene Erlebniffe einflectend, und wiomet bas

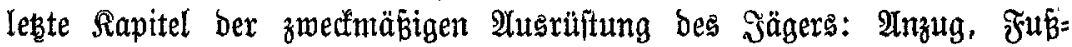

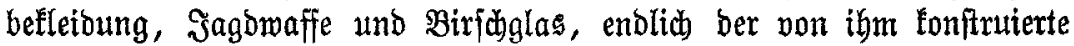
sagbwagen finben furze Sdilderung.

Das anregend geidriebene Büd)lein fann von F̧öriter. Maalter Dber= weiftris in Salenjee bezogen werben.

$\mathrm{F}$.

\section{Nir. 47.}

Bom Jagen, Ixinten unb Rieben. Erinnerungen aus meinem §äger= leben vom "WBilden säger". Mit Mbbildungen von $\mathfrak{B B}$. Arnold, E. Anöllner, Axel Erifsjon und \$hotographien. Eöthen, Ilntalt, 1909. 256 S. Freis eleg. geb. $6,50 \ldots$. Bu beziehen burch Föriter Malter Dhermeiftrib in $\mathfrak{b a l e n j e e ~ b e i ~} \mathfrak{B}$ erlin.

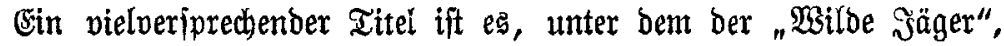

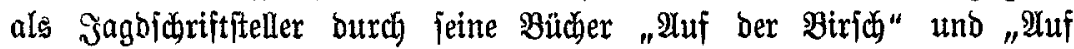

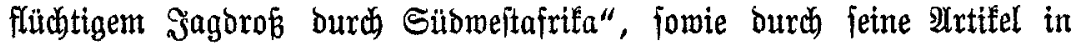

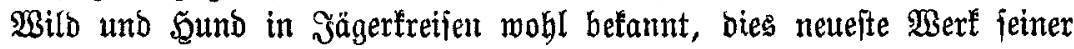
jagblichen Miuje in bie $\mathfrak{W}$ selt jendet, und jebr viel ift in bemjelbent vom Sagen, viel vom Irinfen und wentg (wohl glüctichermeije!) vom

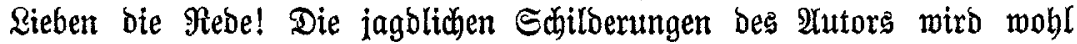

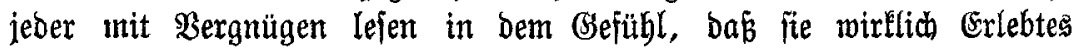
bringen uno von einem Manne gejdrieben fino, der bie Frage, mit was ex fich neben bem eblen $\mathfrak{B e i b w e r t ~ u n c h ~ b e i c h a ̈ f t i g e , ~ h o h n l a ̈ c h e l n d ~ a b m e i ł t , ~}$ und wird babei itber mand berbes $\mathfrak{B}$ sort und über ben itetigen Durit

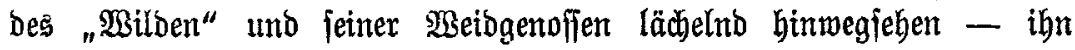
vielleidjt un leteteren uno mebr noch um feine guten Şiridje beneiben! Mit bejonderer Begeifterung - umb jeber, Der jobn vor bem jureienden

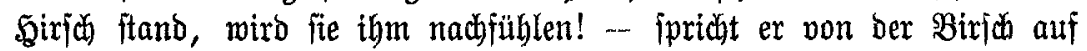
Den itarfen Brunfthiridi); und nidht minder mird man ihm barin recht geber, baß bie Sagd auf ben eblen Uurhabn boch nur bann ihren vollen Reiz gewährt, wenn man fich denfelben jelbit ausmacht uno fich nicht

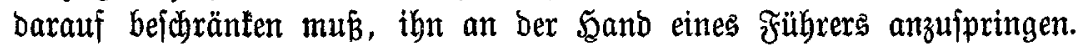
Selbit ift ber Mann - oas gilt vor allent aud beim Metbmert!

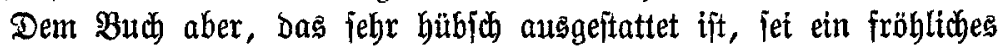
"Weibmannsheil" mit auf Den $\mathfrak{B}$ eg gegeben. 